\title{
Repositions- und Fixationsstößel zur gezielten Kraftappplikation und temporären Fixation von Fragmenten mit Kirschner-Draht
}

\author{
Reto Babst, Matthias Knobe, Frank Beeres, Björn Link
}

Problemstellung: Das Einpassen von Frakturfragmenten, speziell im periartikulären Bereich, erfordert präzise und gezielte Kraftapplikation auf das Fragment [1]. Ein bewährtes Instrument dafür ist der Pfriem. Er erlaubt eine genaue Steuerung des Fragments in den anatomischen Verbund. Ein gutes, ähnlich präzises Repositionsinstrument ist der Zahnarzthaken. Damit lässt sich aber nur geringe Kraft entwickeln. Die scharfe Spitze hat bei beiden den Nachteil, dass sie vor allem im epiphysären oder osteoporotischen Knochen einbricht. Ein konventioneller Stößel kann zwar eine gute Repositionskraft entwickeln und bricht nicht im Knochen ein, ist aber in seiner Repositionswirkung nicht präzise. Allen gemeinsam ist, dass sie das Fragment nur in Position halten und für die Fragmentfixierung ein Handwechsel mit potenziellem Repositionsverlust nötig ist. Zudem kann der Kirschner-Draht nicht genau dort platziert werden, wo die Repositions-

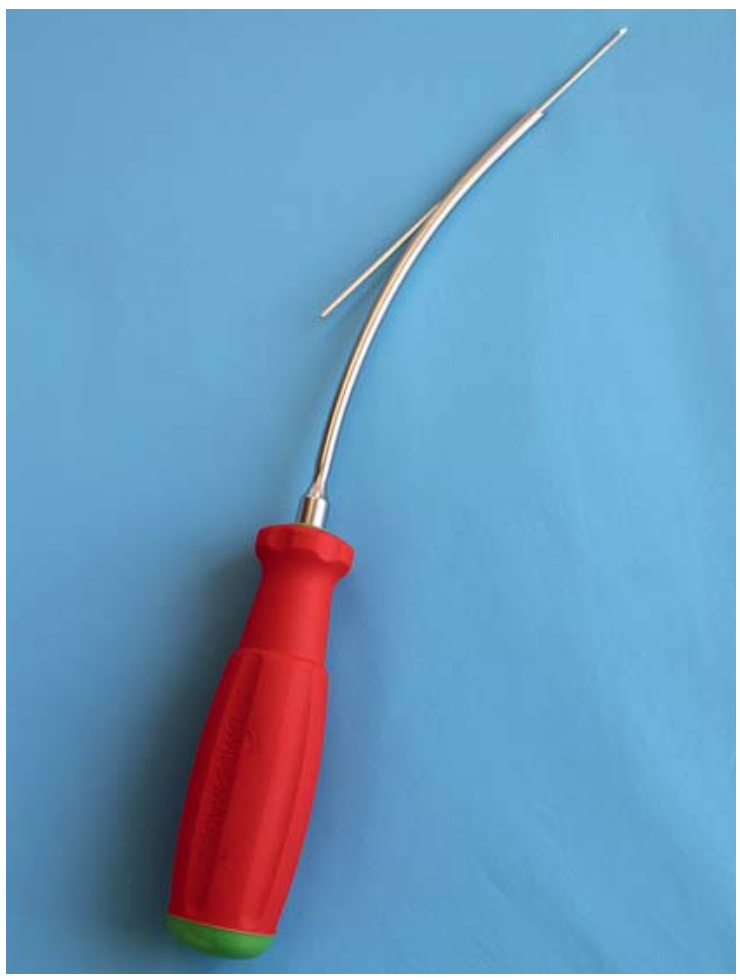

- Abb. 1 Repositions-/Fixationsstößel mit 1,6 mm Kirschner-Draht. kraft appliziert wird, sondern nur neben dem Repositionsinstrument.

Der Repositions-/Fixationsstößel (Fa. Medid, Zürich, nach dem Erstautor benannt) ( $\triangleright$ Abb. 1), erlaubt durch seine Spitze ( $\triangleright$ Abb. 2) die Kraftapplikation dort, wo sie für eine anatomische Reposition notwendig ist, ohne dass ein epi-/metaphysäres oder osteoporotisches Fragment durchbrochen wird ( $\bullet$ Abb. 3 ). Dies wird durch die flächige Auflage verhindert. Bei Erreichen der anatomischen Reposition kann das Fragment mit einem langen Kirschner-Draht (1,6 mm), der im Stößel geführt wird, temporär fixiert werden ( $\triangleright$ Abb.4). Diese Fixation ist ohne einen Handwechsel und damit ohne potenziellen Repositionsverlust möglich. Letzter kann auch durch den Assistenten durch den Stößel eingebracht werden, wobei der Stößel gleichzeitig einen Gewebeschutz darstellt. Die

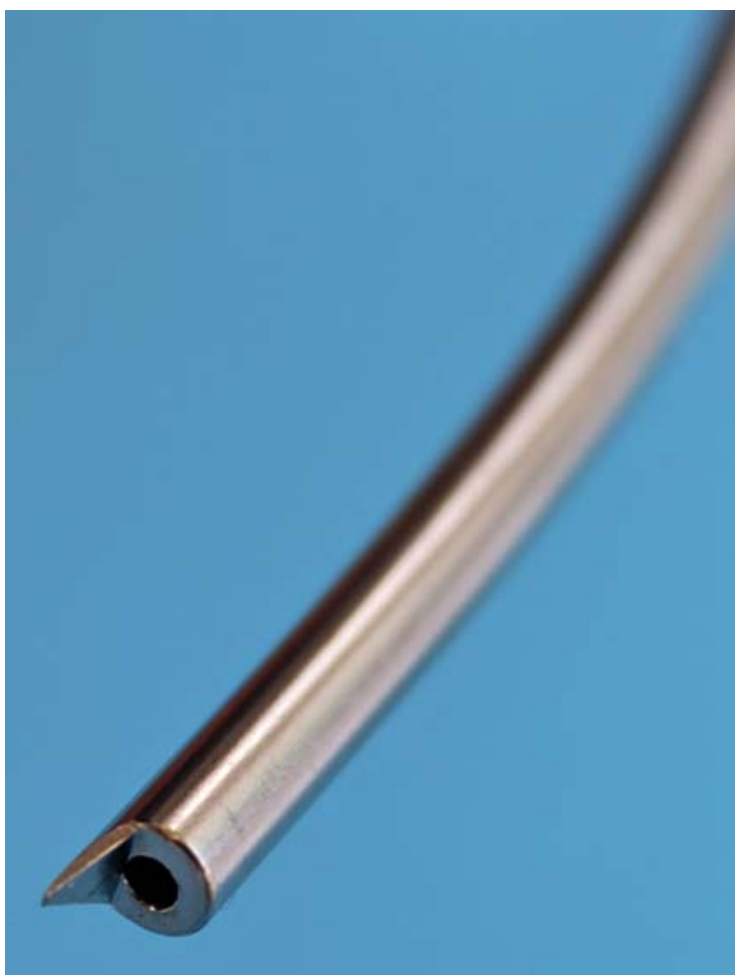

- Abb. 2 Scharfe Spitze, die das Fragment kontrolliert, aber durch die flächige Auflage nicht durch den Knochen bricht. 


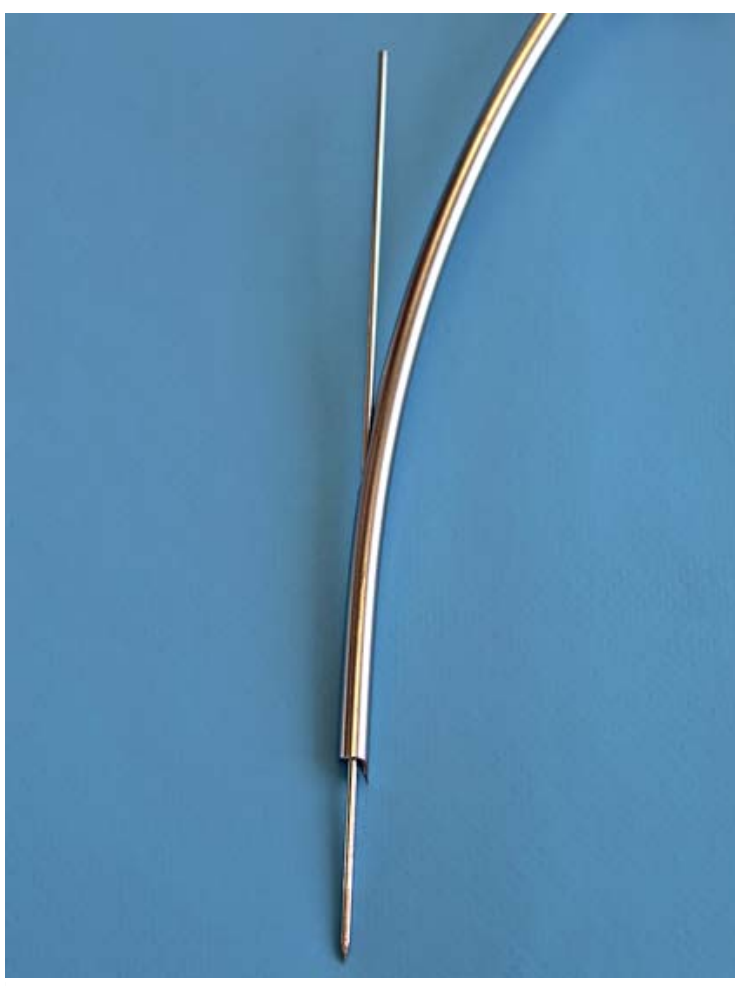

- Abb. 3 Der Repositions-/Fixationsstößel ist gleichzeitig Gewebeschutz für den Kirschner-Draht.

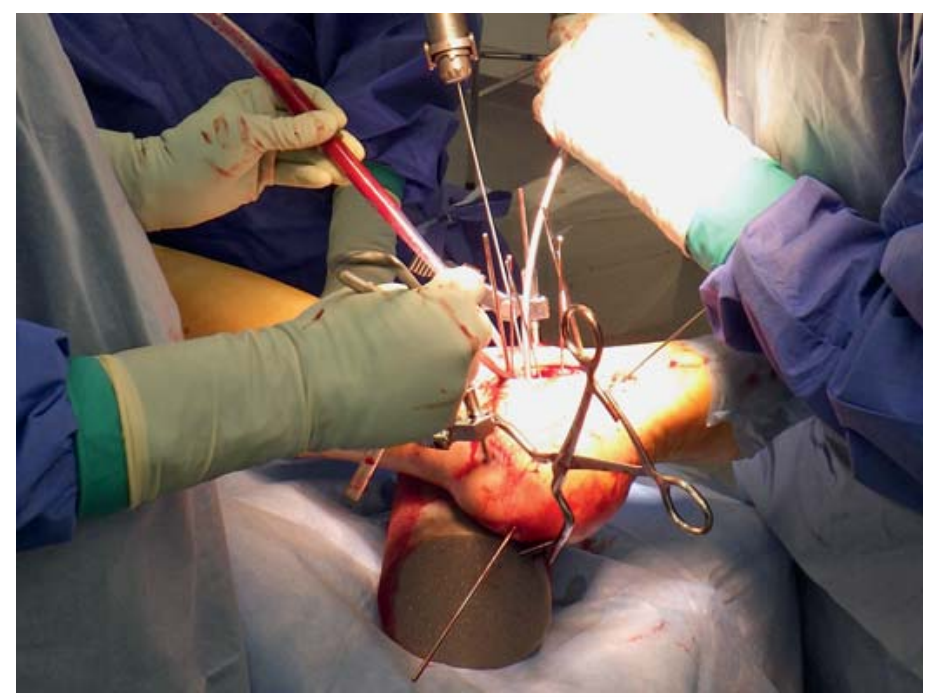

- Abb. 4 Applikation des Repositions-/Fixationsstößels bei einer Kalkaneusfraktur. Der Repositionsstößel ist gleichzeitig Gewebeschutz.
Richtung des Kirschner-Drahtes wird dabei durch den Operateur, der den Stößel bedient, vorgegeben. Zudem ist die temporäre Fixation genau dort platziert, wo die Kraftapplikation durch den Stößel erfolgte.

Schlussfolgerung:

- Der Repositions-/Fixationsstößel erlaubt eine punktuelle Reposition am gewünschten Ort

- keine Knochenperforation trotz relevanter Kraftapplikation

- kein Handwechsel nötig, damit wird ein Repositionsverlust verhindert

- Der Stößel dient gleichzeitig als Gewebeschutz für den eingebrachten temporären Kirschner-Draht, den man durch den Stößel einbringen kann

- Er erlaubt auch perkutan eine präzise Reposition und kann Führungdrähte am gewünschten Ort in der richtigen Richtung unter Schonung des Weichgewebes lenken

Interessenkonflikt

Reto Babst ist Berater der Fa. Medid, Zürich, Schweiz, die anderen Autoren haben keinen Interessenkonflikt.

Literatur

[1] Babst R, Beeres FJP, Link BC. Definitionen und Erklärungen zum Thema Frakturreposition. Unfallchirurg 2019; 122: 88-94

Korrespondenzadresse

Prof. Dr. med. Reto Babst

reto.babst@luks.ch

Bibliografie

DOI https://doi.org/10.1055/a-0950-7186

OP-JOURNAL 2019; 35: 187-188 @ Georg Thieme Verlag KG Stuttgart · New York ISSN 0178-1715 